\title{
Site-specific magnetization reversal studies of magnetite
}

\author{
A. Cady, ${ }^{1}$ D. Haskel, ${ }^{1}$ J. C. Lang, ${ }^{1}$ Z. Islam,,${ }^{1}$ G. Srajer, ${ }^{1}$ A. Ankudinov, ${ }^{2}$ G. Subìas, ${ }^{3}$ and J. Garcìa ${ }^{3}$ \\ ${ }^{1}$ Advanced Photon Source, Argonne National Laboratory, Argonne, Illinois 60439, USA \\ ${ }^{2}$ Department of Physics, University of Washington, Seattle, Washington 98195, USA \\ ${ }^{3}$ Instituto de Ciencia de Materiales de Aragón, CSIC-Universidad de Zaragoza, Pza. San Francisco s/n 50009 Zaragoza, Spain \\ (Received 7 November 2005; revised manuscript received 17 February 2006; published 14 April 2006; corrected 20 April 2006)
}

\begin{abstract}
The mechanism of magnetization reversal in magnetite $\left(\mathrm{Fe}_{3} \mathrm{O}_{4}\right)$ single crystals was studied using sitespecific magnetic sensitive diffraction anomalous near-edge structure. By exploiting the angular dependence of the cross section, we are able to show that the mechanism of reversal involves a mixture of coherent rotation and domain formation. The results reveal additional details to that provided by XMCD measurements, which average over nonequivalent sites.
\end{abstract}

DOI: 10.1103/PhysRevB.73.144416

PACS number(s): 75.60.-d, 75.25.+z, 91.60.Pn, 61.10.Eq

\section{INTRODUCTION}

Magnetite, one of the oldest known magnetic materials, has remained the subject of extensive experimental and theoretical investigation owing largely to its low temperature metal-to-insulator transition, i.e., the Verwey $\operatorname{transition}^{1}$ at $T \approx 120 \mathrm{~K}$, as well as its potential in technological applications. The crystal structure of magnetite has eight formula units $\left(\mathrm{Fe}_{3} \mathrm{O}_{4}\right)$ arranged in a cubic lattice in which the $\mathrm{Fe}$ ions reside on eight tetrahedral sites and 16 octahedral sites. To date, numerous studies have focused on the nature of the metal-to-insulator transition and whether it is explained by charge ordering on the octahedral sites, as postulated by Verwey. Verwey's hypothesis is still debated. ${ }^{2-5}$ Subsequently, magnetite has been subjected to many new experimental probes, including the study of site-specific phonon density of states, ${ }^{6}$ site-specific atomic imaging, ${ }^{7}$ and site-specific diffraction anomalous near-edge structure (DANES) studies. ${ }^{8-10}$ These types of site-specific measurements may greatly advance our understanding of magnetite and help resolve some still debated issues.

It is widely accepted that magnetite is a ferrimagnetic material, the magnetic moments at the tetrahedral sites being antialigned with the moments at the octahedral sites. ${ }^{11}$ Its magnetic easy axis along the [111] direction changes to the [001] direction below the Verwey transition. ${ }^{12}$ At room temperature, the [001] axis is the hard axis, and the [110] axes are intermediate. In bulk crystals of magnetite, these axes are determined by the material's magnetocrystalline anisotropy (MCA). The MCA associated with each of the two nonequivalent sites should, when combined, yield the [111] easy axis and determine the intrinsic field reversal properties of magnetite. In $\mathrm{Nd}_{2} \mathrm{Fe}_{14} \mathrm{~B}$, it was found, for instance, that the [001] easy axis of magnetization arises predominantly from one of two nonequivalent sites. ${ }^{13}$ Previous researchers have performed site-specific x-ray magnetic circular dichroism on ferrite powders ${ }^{14}$ and crystals, ${ }^{15}$ but to our knowledge, measurements have not been performed to examine how the magnetic moments of octahedral and tetrahedral sites in crystals of magnetite individually respond to reversal of applied magnetic field. We have performed magnetic-sensitive DANES studies at Bragg reflections that separate the non- equivalent sites to obtain site-specific magnetic field-reversal loops. The results indicate differences between the sites, e.g., coercivity, but demonstrate that each site, taken alone, reverses in a way that is consistent with the [111] direction as the easy axis. The results also show that the magnetic reversal mechanism is a combination of coherent rotation of the magnetic moments and domain formation. In the following section, we describe the experimental setup and technique. We present the results in Sec. III, followed by discussion and interpretation of the results in Sec. IV and a brief conclusion in Sec. V.

\section{EXPERIMENTAL SETUP AND TECHNIQUE}

The nonequivalent sites were isolated by selection of appropriate Bragg reflections, e.g., the structure factor at the (222) peak contains only contributions from the octahedral sites while the (022) peak arises only from the tetrahedral Fe sites. ${ }^{10}$ By measuring the asymmetry in the DANES at these two reflections for opposite helicity of circularly polarized $\mathrm{x}$ rays, one can retrieve magnetic contributions of the sites separately. This is the magnetic analogy of DANES, i.e., MDANES. ${ }^{13}$ Magnetic reversal properties can be obtained by varying an applied magnetic field and measuring the induced changes at each reflection. The results are analogous to site-specific hysteresis loops.

The measurements were performed at undulator beamline 4-ID-D at the Advanced Photon Source on single crystals of magnetite whose [011] or [111] directions were oriented along the scattering vector as illustrated in Fig. 1. In both crystals, cut from a common crystal of magnetite, an electromagnet delivered a magnetic field ( $\leqslant 6 \mathrm{kOe})$ along the [011] (or equivalent) direction, which was oriented parallel to the sample surface and in the scattering plane. Beamline 4-ID-D makes use of a diamond (111) phase retarder operated in Bragg transmission geometry to convert the x-ray polarization from linear to circular. ${ }^{16}$ The phase retarder is driven by a piezoactuator to rapidly switch between opposite $\mathrm{x}$-ray helicities. The MDANES spectrum was measured through the Fe $K$-edge resonance using a digital lock-in detection scheme that synchronizes measurement of the incident and diffracted intensity with the helicity modulation $(1 \mathrm{~Hz})$ of the incoming 
a)

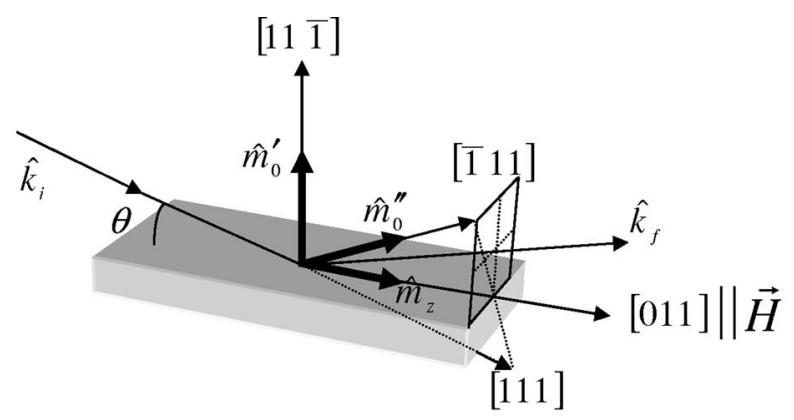

b)

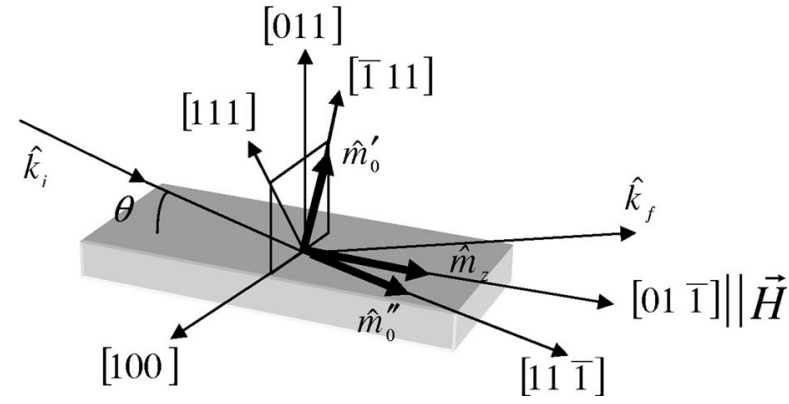

FIG. 1. Orientations of the two samples studied relative to the scattering plane. The slabs represent the single crystal samples, where $\hat{k}_{i}$ and $\hat{k}_{f}$ are the incident and scattered wave vectors. The magnetic field $(\vec{H})$ is applied along the [011] and [01 $\overline{1}]$ directions in (a) and (b), respectively. Reciprocal space directions relevant to the discussion are labelled and possible remanent magnetic moment directions considered $\left(\hat{m}_{0}^{\prime}\right.$ and $\left.\hat{m}_{0}^{\prime \prime}\right)$ are shown, as well as the magnetic moment $\left(\hat{m}_{z}\right)$ representing the saturation condition. The $\hat{y}$ direction is along the sample normal, and $(\vec{H})$ is applied along $\hat{z}$.

X-ray beam. A dual photon counter measures the incident intensity from an ion chamber and diffracted intensity from an avalanche photodiode signal over many cycles of helicity switching for each incident energy. The Si (111) doublecrystal monochromator, phase retarder, and sample's Bragg angles were tracked to maintain their respective fixed- $Q$ conditions through the resonance scans. The sample's absorption and dichroism were measured in the fluorescence channel concomitantly with the MDANES. All the measurements were performed at room temperature.

To understand the origin of the MDANES signal, one must consider the form factor associated with the magnetic ions. Neglecting nonresonant magnetic scattering and linear dichroism terms, the electric dipole scattering amplitude from a single magnetic ion near resonance is given by $f$ $=f_{e}(Q, E)\left(\hat{\boldsymbol{\epsilon}}^{\prime} \cdot \hat{\boldsymbol{\epsilon}}\right)-i f_{m}(E)\left(\hat{\boldsymbol{\epsilon}}^{\prime} \times \hat{\boldsymbol{\epsilon}}\right) \cdot \hat{m} .{ }^{17}$ The term $f_{e}$ includes nonresonant and resonant charge scattering while $f_{m}$ is purely resonant magnetic scattering. $\epsilon$ and $\epsilon^{\prime}$ are the polarizations of the incident and scattered $\mathrm{x}$ rays, respectively. $\hat{m}$ denotes the magnetic moment direction. In the kinematic scattering approximation, the scattered intensity is then related to the structure factor as $|F(Q, E)|^{2}=\left|\Sigma_{n} f_{n} e^{i \vec{Q} \cdot \vec{r}_{i}}\right|^{2}$, where the sum is over all atoms in the unit cell. In the results section, we show that the kinematic approximation is appropriate in this case. Since reversing x-ray helicity is equivalent to reversing magnetization direction, a differential measurement of diffracted intensity for opposite x-ray helicities $\left(I^{+}-I^{-}\right)$removes pure charge terms $\left(f_{e}^{*} f_{e}\right.$, independent of $\left.\hat{m}\right)$

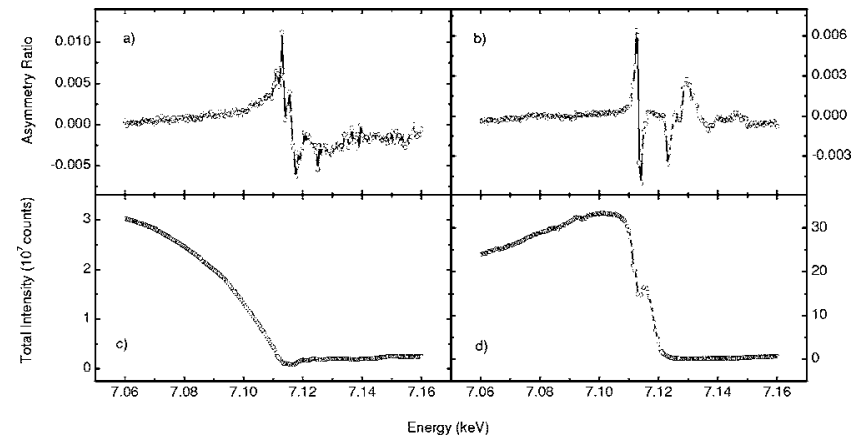

FIG. 2. The AR or MDANES as a function of incident $x$-ray energy is shown in (a) and (b) as measured in the (222) and (022) diffraction conditions, respectively. A 6 kOe magnetic field was applied to the sample during the data acquisition. Note that the relative signs of the MDANES at the two sites depends on energy. Also shown in (c) and (d) are the corresponding measurements of the total scattered intensity or DANES as a function of incident $\mathrm{x}$-ray energy as measured in the (222) and (022) diffraction conditions, respectively.

and pure magnetic-sensitive terms $\left(f_{m}^{*} f_{m}\right.$, quadratic in $\left.\hat{m}\right)$, and includes contributions from charge-magnetic interference terms only, i.e., $\left(-i \sum_{i, j} A^{*} B_{j} f_{e, i}^{*} f_{m, j} e^{i \vec{Q} \cdot\left(\vec{r}_{i}-\vec{r}_{j}\right)}+\right.$ c.c. $)$, proportional to $\hat{m}$ ( $A$ and $B$ are polarization factors). Adding diffracted intensities for opposite $\mathrm{X}$-ray helicities $\left(\mathrm{I}^{+}+\mathrm{I}^{-}\right)$ removes charge-magnetic interference scattering and practically measures resonant charge scattering since the pure magnetic scattering is very small. The MDANES signal is recorded as an asymmetry ratio (AR) quantified as the difference in diffracted intensities normalized by the sum $\mathrm{AR}=\left(I^{+}-I^{-}\right) /\left(I^{+}+I^{-}\right)$. The AR signal thus provides magnetic information about the structure. Specifically, it measures a projection of the magnetic moment along the incident $\left(\hat{k}_{i}\right)$ and scattered $\left(\hat{k}_{f}\right)$ wave vectors that has been derived to be $\left[\left(\hat{k}_{i} \cdot \hat{m}\right)+\left(\hat{k}_{f} \cdot \hat{m}\right) \cos 2 \theta\right]$ (Refs. 13 and 18) with $\theta$ the Bragg angle. In contrast, XMCD (Ref. 19) measures the magnetic moment's projection along $\hat{k}_{i}$.

\section{RESULTS}

Two types of measurements are presented in this paper: (1) MDANES through the Fe $K$-edge resonance and (2) its response to a varying magnetic field at a selected resonant energy. Figure 2 presents MDANES measurements at the (222) and (022) reflections. Clearly, the MDANES spectra are different for the two sites. As shown in Fig. 2, the DANES spectra are dominated by self-absorption above the Fe $K$-edge $(7.112 \mathrm{keV})$. Despite this fact, the MDANES yields a reliable magnetic signal in this region owing to the measurement's differential nature and the lock-in approach. Since the MDANES contains both chemical and magnetic information, these differences in the MDANES for the two sites do not necessarily arise from different magnetic properties of the two sites. The data presented in Fig. 2 are the averages of many separate data sets taken under equivalent experimental conditions. 


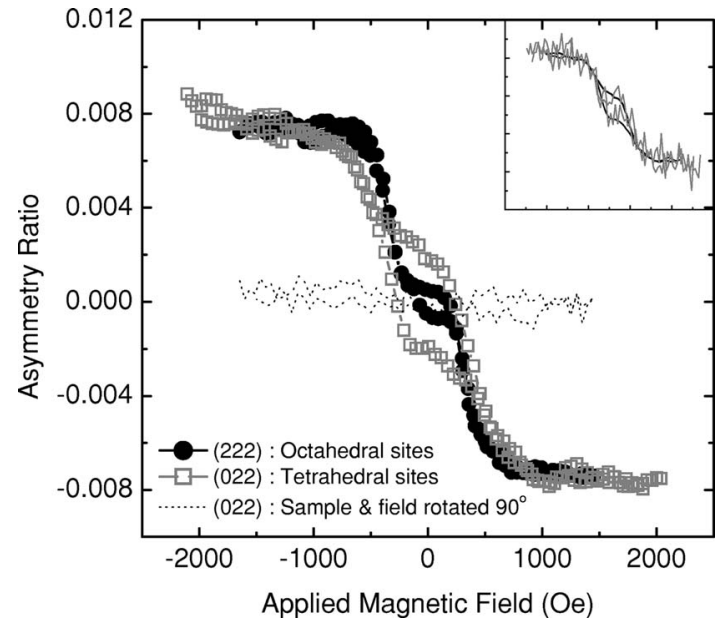

FIG. 3. The magnetic sensitive AR plotted as a function of applied magnetic field as measured in the (222) (black) and the (022) (gray) diffraction conditions. The AR was measured with incident x-ray energy of $7.112 \mathrm{keV}$ where the AR magnitude was maximum. The error bars are comparable to the symbol sizes. The AR measured at the (022) reflection with both the magnet and sample rotated 90 deg to the scattering plane is shown by the dashed line and, as described in the text, indicates the absence of any net magnetization transverse to the field direction throughout the reversal. The inset shows the agreement between AR measured in the fluorescence channel (gray line) and a weighted average of the site-specific hysteresis loops (black line).

Figure 3 presents field reversal loops measured at the (022) and (222) reflections. The loops are clearly different at the two Bragg reflections. At the (022) reflection (tetrahedral sites), there is a significant hysteresis as a function of field. At the (222) reflection (octahedral sites), there is a much smaller hysteresis, and the magnetization plateaus more in the small applied field region. Unlike the MDANES signal, which contains chemical information, these different loops do by themselves indicate a different magnetic response for the two sites.

In the inset of Fig. 3, we have shown the consistency of the concomitant fluorescence measurement (gray line) with a weighted average of the site-specific hysteresis loops (black line). ${ }^{20}$ It is also important to point out that independent measurements of XMCD in transmission through powder magnetite samples follow the same general slope of the fluorescence and MDANES measurements, but do not show the plateau structure in the small field region of the loops presented in Fig. 3. When each of the samples and the magnet were rotated by $90 \mathrm{deg}$, there was no measurable AR throughout the field reversal, indicating there was no net magnetization in the transverse direction during the reversal process. The AR measurement taken in this condition for the (022) reflection is shown by the dashed line in Fig. 3. The equivalent measurement in the (222) reflection is also zero throughout this field range.

In the preceding section, we claimed that the kinematic scattering approximation was a valid approximation in our case. This is because we are measuring differences and relative magnitudes for a given reflection, and the extinction effects are exactly the same upon helicity reversal of the light
TABLE I. The angular dependence of AR $(\Phi)$ calculated for the octahedral $\left(O_{h}\right)$ and tetrahedral $\left(T_{h}\right)$ sites under the conditions of magnetic saturation along $\hat{m}_{z}$ or remanence along either $\hat{m}_{0}^{\prime}$ or $\hat{m}_{0}^{\prime \prime}$. The calculated remanence-to-saturation ratios $(R)$ are compared to the measured values, $R_{\text {exp }}$.

\begin{tabular}{ccccc}
\hline \hline & $\Phi_{\hat{m}_{z}}$ & $\Phi_{\hat{m}_{0}^{\prime}}$ & $\Phi_{\hat{m}_{0}^{\prime \prime}}$ & $R_{\text {exp }}$ \\
\hline$O_{h}(222)$ & 1.76 & $-0.04(R=-0.02)$ & $1.52(R=0.86)$ & 0.07 \\
$T_{d}(022)$ & 1.75 & $-0.04(R=-0.02)$ & $1.40(R=0.80)$ & 0.31 \\
\hline \hline
\end{tabular}

or upon changing an applied magnetic field. This can be seen in Fig. 3, where the difference signal is shown for the case where the magnetic field is applied perpendicular to the scattering plane. The asymmetry ratio vanishes, as expected from the scattering cross section, but also indicating that extinction effects, even if present, do not depend on helicity. The site-specific dichroic effect is verified since the average corresponds with the dichroism measured in fluorescence as pointed out in Fig. 3. Furthermore, strong anomalies such as spurious peaks due to multiple reflections with the same Bragg angle are absent in the DANES plots.

\section{DISCUSSION}

As shown in Fig. 3, the hysteresis loops for the two sites in magnetite are different. The one for the octahedral sites is entirely enclosed by that associated with the tetrahedral sites, indicating that tetrahedral sites may exhibit a larger coercivity. The difference in the remanence-to-saturation ratios is more apparent. By fitting the central portion of the fieldreversal loops (between $\approx-250$ and $250 \mathrm{Oe}$ ) to lines, we determined the AR corresponding to the remanent magnetization values on both increasing and decreasing fields. Dividing the difference by the saturation value of $A R$, we obtained $\approx 0.07$ for the octahedral sites and $\approx 0.31$ for the tetrahedral sites. To understand this difference, we must consider the angular dependence of the scattering cross section in more detail. Using the diagrams in Fig. 1 as a guide, we have calculated what the expected AR signal would be if the magnetization vector lay along different crystal directions. The results, summarized in Table I, show that the magnetic reversal mechanism is a combination of coherent rotation and domain formation.

With the full $6 \mathrm{kOe}$ field applied along the [011] direction (or [01] for the (022) reflection), corresponding to an intermediate magnetic axis, the magnetization is saturated as represented by $\hat{m}_{z}$ in Fig. 1 In this case, we measure the AR corresponding to the saturated parts of the loops plotted in Fig. 3. Defining $\hat{k}_{i}=-\sin \theta \hat{y}+\cos \theta \hat{z}, \hat{k}_{f}=\sin \theta \hat{y}+\cos \theta \hat{z}$, and $\hat{m}=\hat{z}$, we obtain for the angular dependence of AR: $\Phi(\mathrm{AR})$ $=\left(\hat{k}_{i} \cdot \hat{m}\right)+\left(\hat{k}_{f} \cdot \hat{m}\right) \cos 2 \theta=1.75$ for the tetrahedral sites and 1.76 for the octahedral sites where we have used the Bragg angles $\theta$ of 17 and $21 \mathrm{deg}$ for the (022) and (222) reflections, respectively.

As the magnetic field is reversed, the magnetization is reduced, and a smaller AR signal is measured. We consider several scenarios for the direction of the remanent magneti- 
zation that give rise to the nonzero AR at $H=0$. The simplest case is that the magnetization at each site rotates to a magnetic easy axis (the [111] or equivalent directions) as the applied field is reduced through zero. Possible magnetization directions along easy axes are shown by $\hat{m}_{0}^{\prime \prime}$ and $\hat{m}_{0}^{\prime}$ in Figs. 1(a) and 1(b). According to the previously defined $\Phi(\mathrm{AR})$, one can calculate the expected ratio of AR with $\hat{m}$ along one of the easy axes to that of AR along the saturation direction. First, tetrahedral sites are considered assuming that these sites are magnetized along the [11 $\overline{1}]$ easy axis at $H=0$, labeled by $\hat{m}_{0}^{\prime \prime}$ in Fig. 1(b), and the (022) reflection is probed. Then, the angular dependence of AR becomes 1.40. The resulting remanence-to-saturation ratio would be approximately 0.80 . If the remanent magnetization goes to the [ $\overline{1} 11]$ easy axis, labeled by $\hat{m}_{0}^{\prime}$ in Fig. 1(b), the angular dependence gives $\mathrm{AR}=-0.04$, and the remanence-to-saturation ratio becomes -0.02 . The experimental ratio is 0.31 , which is between these two values. The results of the same exercise for octahedral sites are also shown in Table I. As determined from hysteresis loop measurements with the sample and a magnetic field direction at $90 \mathrm{deg}$ to the incident beam, there is no component of magnetization in the transverse direction. This indicates that if the magnetization is along the easy axis $\hat{m}_{0}^{\prime \prime}$ or $\hat{m}_{0}^{\prime}$ as shown in Fig. 1(b) or along $\hat{m}_{0}^{\prime \prime}$ in Fig. 1(a), then there must be domains such that there is an equal probability, for instance, of the magnetization lying along [111] and [111] in Fig. 1(b). The component of magnetization perpendicular to the scattering plane would then be zero, consistent with the transverse field reversal measurement.

One could argue, based on the ratio of remanence-tosaturation magnetization in the hysteresis loops of 0.31 for the tetrahedral sites, that the magnetization lies on average between the $\hat{m}_{0}^{\prime}$ (ratio of -0.02 ) and $\hat{m}_{0}^{\prime \prime}$ directions (ratio of 0.8 ) in Fig. 1(b) if a coherent reversal process is assumed. The ratio associated with the octahedral site $(0.07)$ could be explained by most of the magnetization being aligned along $\hat{m}_{0}^{\prime}$. However, as we have demonstrated the absence of a net magnetization component in the plane perpendicular to the applied field direction, a purely coherent reversal process can be ruled out for the case of tetrahedral sites (but not for octahedral sites), as such a process would have resulted in nonzero net magnetization transverse to the applied field.
Similarly, a pure domain nucleation and growth process without coherent rotation cannot explain the data either. While such a process would yield a null AR at $90 \mathrm{deg}$ (domains are magnetized along the applied field and perpendicular to the scattering plane), the remanence-to-saturation ratios expected for such a process are nearly the same for both reflections, as both reflections have similar Bragg angles and both average over the same ensemble of domains with opposite $\hat{z}$ orientations. This leads us to conclude that the mechanism of reversal must be a combination of coherent rotation and domain nucleation and growth.

\section{CONCLUSION}

We have presented site-specific magnetism studies on magnetite that demonstrate the capabilities of magnetic sensitive diffraction anomalous near-edge structures to resolve the reversal of magnetic moments associated with dissimilar local atomic environments. The results may indicate some differences in the magnetic reversal behavior for the tetrahedral and octahedral sites in magnetite and also show that the reversal most likely occurs partially through domain formation and partially through coherent rotation of the magnetization. In the future it would be interesting to continue these studies (with advancements in experimental capability) at low temperature. This might provide some insight into the still-debated nature of the Verwey transition. In addition, it would be desirable to examine the site-specific phenomena in films to determine how stress anisotropy affects the reversal. First-principles calculations have predicted that under extensive lateral strain the tetrahedral $\mathrm{Fe}$ sites would favor magnetization perpendicular to that of the octahedral $\mathrm{Fe}$ sites. ${ }^{21}$ In principle, such theories could be tested using the approach described here.

\section{ACKNOWLEDGMENTS}

We thank J. W. Freeland, E. Kravtsov, and D. R. Lee for useful discussions of the results. Work at the Advanced Photon Source was supported by the U.S. Department of Energy, Office of Basic Sciences, under Contract No. W-31-109ENG-38.
${ }^{1}$ E. J. W. Verwey and P. W. Haayman, Physica (Amsterdam) 8, 979 (1941); E. J. W. Verwey, P. W. Haayman, and F. C. Romeijn, J. Chem. Phys. 15, 181 (1947).

${ }^{2}$ H.-T. Jeng, G. Y. Guo, and D. J. Huang, Phys. Rev. Lett. 93, 156403 (2004).

${ }^{3}$ J. Garcìa and G. Subìas, J. Phys.: Condens. Matter 16, R145 (2004).

${ }^{4}$ J. P. Wright, J. P. Attfield, and P. G. Radaelli, Phys. Rev. Lett. 87, 266401 (2001).

${ }^{5}$ I. Leonov, A. N. Yaresko, V. N. Antonov, M. A. Korotin, and V. I. Anisimov, Phys. Rev. Lett. 93, 146404 (2004).

${ }^{6}$ M. Seto, S. Kitao, Y. Kobayashi, R. Haruki, Y. Yoda, T. Mitsui, and T. Ishikawa, Phys. Rev. Lett. 91, 185505 (2003).

${ }^{7}$ P. Korecki, M. Szymoński, J. Korecki, and T. Ślęzak, Phys. Rev. Lett. 92, 205501 (2004).

${ }^{8}$ H. Stragier, J. O. Cross, J. J. Rehr, L. B. Sorensen, C. E. Bouldin, and J. C. Woicik, Phys. Rev. Lett. 69, 3064 (1992).

${ }^{9}$ G. Subìas, J. Garcìa, J. Blasco, M. Grazia Proietti, H. Renevier, and M. Concepcion Sánchez, Phys. Rev. Lett. 93, 156408 (2004).

${ }^{10}$ A. I. Frenkel, J. O. Cross, D. M. Fanning, and I. K. Robinson, J. Synchrotron Radiat. 6, 332 (1999).

${ }^{11}$ C. Kittel, Introduction to Solid State Physics, 7th ed. (Wiley, New York, 1996). 
${ }^{12}$ C. Medrano, M. Schlenker, J. Baruchel, J. Espeso, and Y. Miyamoto, Phys. Rev. B 59, 1185 (1999).

${ }^{13}$ D. Haskel, J. C. Lang, Z. Islam, A. Cady, G. Srajer, M. van Veenendaal, and P. C. Canfield, Phys. Rev. Lett. 95, 217207 (2005); D. Haskel, J. Lang, Z. Islam, G. Srajer, J. Cross, and P. Canfield, IEEE Trans. Magn. 40, 2874 (2004).

${ }^{14}$ K. Matsumoto, F. Saito, T. Toyoda, K. Ohkubo, K. Yamawaki, T. Mori, K. Hirano, M. Tanaka, and S. Sasaki, Jpn. J. Appl. Phys., Part 1 39, 6089 (2000).

${ }^{15}$ K. Kobayashi, H. Kawata, and K. Mori, J. Synchrotron Radiat. 5, 972 (1998).

${ }^{16}$ J. C. Lang and G. Srajer, Rev. Sci. Instrum. 66, 1540 (1995).

${ }^{17}$ J. P. Hannon, G. T. Trammell, M. Blume, and D. Gibbs, Phys. Rev. Lett. 61, 1245 (1988).

${ }^{18}$ D. Haskel, G. Srajer, J. C. Lang, J. Pollmann, C. S. Nelson, J. S.
Jiang, and S. D. Bader, Phys. Rev. Lett. 87, 207201 (2001). Note that the $\cos 2 \theta$ term was misplaced in this reference and similarly in Ref. 13.

${ }^{19}$ J. Stöhr, J. Magn. Magn. Mater. 200, 470 (1999).

${ }^{20}$ The proper weighted average was obtained using FEFF8 calculations, showing that at $E=7.112 \mathrm{keV}$, the XMCD contributions from tetrahedral sites is six times greater than the contributions from octahedral sites. Since there are twice as many $O_{h}$ sites as $T_{d}$ sites, we used the weighted average $2 / 3\left[O_{h}\right]+6 / 3\left[T_{d}\right]$, where $\left[O_{h}\right]$ and $\left[T_{d}\right]$ represent the hysteresis loops obtained from the octahedral and tetrahedral sites, respectively. We have used addition because the two sites yield the same sign of XMCD signal at this energy, despite their opposite magnetic moments.

${ }^{21}$ H.-T. Jeng and G. Y. Guo, Phys. Rev. B 65, 094429 (2002). 